\title{
In memoria di Francesco Orlando
}

\section{Francesco Fiorentino}

\section{(2) OpenEdition}

\section{Journals}

\section{Edizione digitale}

URL: http://journals.openedition.org/studifrancesi/5837

DOI: 10.4000/studifrancesi.5837

ISSN: 2421-5856

\section{Editore}

Rosenberg \& Sellier

\section{Edizione cartacea}

Data di pubblicazione: 1 mai 2011

Paginazione: 138-140

ISSN: 0039-2944

\section{Notizia bibliografica digitale}

Francesco Fiorentino, «In memoria di Francesco Orlando», Studi Francesi [Online], 163 (LV | I) | 2011 online dal 30 novembre 2015, consultato il 13 janvier 2021. URL: http://journals.openedition.org/ studifrancesi/5837 ; DOI: https://doi.org/10.4000/studifrancesi.5837

\section{(c) (i) (9)}

Studi Francesi è distribuita con Licenza Creative Commons Attribuzione - Non commerciale - Non opere derivate 4.0 Internazionale. 


\section{In memoria di Francesco Orlando}

Francesco Orlando è nato a Palermo nel 1934 da una famiglia di antica borghesia che poteva vantare fra i suoi membri avvocati illustri e il Presidente del Consiglio della vittoria del 1918, Vittorio Emanuele Orlando. La sua vocazione letteraria fu precocissima. Lettore infaticabile dalla memoria prodigiosa, già da bambino, a tredici anni, si era cimentato nella traduzione del teatro di Victor Hugo. Neppure gli studi di Giurisprudenza, intrapresi in ossequio alla tradizione familiare, ebbero il potere di distoglierlo dalla letteratura. Tanto più in quanto in quegli anni avveniva l'incontro decisivo nella sua formazione, quello con Giuseppe Tomasi di Lampedusa che lo elesse a suo allievo, prima esclusivo poi all'interno di una ristrettissima cerchia, delle sue lezioni di letteratura francese e di letteratura inglese, oggi pubblicate in volume nella collana dei «Meridiani». Su quest'incontro Orlando è tornato in un prezioso racconto autobiografico che ha avuto ben due versioni: la prima nel 1963 presso Scheiwiller; la seconda, con una fondamentale aggiunta, presso Bollati nel 1996. In quest'ultima, con spregiudicatezza rousseauiana (Rousseau è stato uno dei suoi autori prediletti), egli ha saputo indagare anche gli equivoci che caratterizzarono il rapporto con il maestro. L'insegnamento dell'autore del Gattopardo ha segnato in maniera indelebile la personalità di studioso di Orlando. Gli ha lasciato in eredità quella curiosità inesauribile del dilettante coltissimo che ripugna alle esclusività dello specialismo, la disinvoltura del gran signore delle lettere che si sente a casa sua ovunque, nell'antichità classica, nella letteratura bizantina come in quella d'avanguardia. Questa passione letteraria (e musicale) onnivora, coltivata da chi non ha mai fatto entrare la televisione in casa propria, e ha potuto così dedicare la maggior parte delle sue serate alla lettura, si accompagnava sempre al rigore inflessibile del teorico.

La partenza da Palermo, città con cui intrattenne un rapporto contraddittorio ma sempre intensissimo, per Pisa, dove, salvo un breve intervallo veneziano, avrà casa fino alla morte, segnò l'inizio ufficiale della sua carriera di studioso di letteratura francese. Qui fu accolto da Arnaldo Pizzorusso che guidò l'inizio dei suoi studi con un lavoro di tesi su Ramond de Carbonnières, scrittore rousseauiano di paesaggi alpestri: studio, che ora sta per apparire in Francia, dove già si annunzia una prospettiva innovativa nell'interpretazione della svolta di fine Settecento. L'altro grande centro dei suoi interessi è costituito dal teatro del Seicento. Nel libro dedicato a Rotrou (1963), autore allora ancora trascurato, si sente l'influsso dei lavori di Jean Rousset sul barocco che combinano analisi tematica e prospettiva storiografica. Segue un saggio che ha lasciato il segno negli studi francesistici: Infanzia, memoria e storia da Rousseau ai romantici (1966, ristampato nel 2007) in cui, attraverso il tema del ricordo d'infanzia in vari autori di fine Sette e inizi Ottocento, vengono ricostruiti i termini di una svolta capitale nella sensibilità e nella letteratura francese. Già in questa prima fase egli elegge a punto di riferimento dei suoi studi Erich Auerbach, alla cui lezione resterà sempre fedele. Nel 1969 vince la cattedra di Letteratura francese presso la Facoltà di Lingue e Letterature Straniere di Pisa. Erano anni incandescenti per l'università italiana. Orlando, animato come in tutta la vita da passione civile, vede nella rivolta 
studentesca del Sessantotto una grande possibilità di rinnovamento della cultura italiana e di liberazione dei costumi da pregiudizi e condizionamenti autoritari. Paga il suo impegno con la cancellazione dell'incarico alla Scuola Normale di Pisa e deciderà di trasferire il proprio insegnamento prima a Napoli e poi a Venezia. Insegnamento che ovunque conoscerà un successo incredibile giacché egli sapeva, come pochi, affascinare i giovani con la parola e con l'esempio. In seguito, nel 1982, tornerà a insegnare a Pisa, città adorata, prima alla Facoltà di Lingue, poi a quella di Lettere.

Continuerà a interessarsi di letteratura francese fino alla fine della sua vita, come testimoniano sia i saggi raccolti nel volume Le costanti e le varianti (il Mulino, 1983), sia soprattutto il volume Illuminismo, Barocco e retorica freudiana (1982 e 1997) che contiene una magistrale analisi del passaggio nella cultura francese - oltre che europea - dal codice letterario barocco dominato dalla metafora a quello illuministico incentrato sull'ironia. Tuttavia, a partire dalla fine degli anni Sessanta, ai suoi interessi francesistici si stavano affiancando quelli di teoria letteraria. Erano gli anni dello strutturalismo, anni in cui la critica italiana stava profondamente innovandosi sotto l'influsso delle correnti critiche d'oltralpe. Orlando, pur facendo sua la lezione della linguistica strutturale (Saussure e Hjelmslev sono i linguisti a cui si riferisce) e soprattutto della psicanalisi, assume una posizione originale nel panorama degli studi non solo italiani. In particolare privilegia della lezione freudiana gli aspetti linguistici (l'opera capitale del maestro di Vienna è per lui Il motto di spirito e $i$ suoi rapporti con l'inconscio, a cui dedicherà un'importante introduzione nell'edizione Boringhieri del 1975) a scapito degli usi, fino ad allora dominanti, che erano rimasti fedeli a una concezione psicologistica e contenutistica (se non addirittura simbolica) dell'interpretazione e che inevitabilmente, nel loro tentativo di ricostruzione dell'inconscio dell'autore, erano votati a una deriva biografistica. Lettura freudiana della "Phèdre", apparso nel 1971, è il primo volume di una tetralogia (sempre einaudiana) in cui si combinano speculazioni e analisi illuminanti di capolavori come Fedra, Misantropo, Lettere persiane, in grazia delle quali Orlando intende costruire una vera e propria proposta organica di teoria della letteratura. Si tratta di un'operazione che per vastità, coerenza e originalità non ha eguali nella cultura italiana, e non solo. Una proposta teorica, basata sulla nozione freudiana di formazione di compromesso, che rende possibile riconoscere sia sul piano formale che su quello contenutistico del testo letterario un sistema di tensioni fra istanze diverse e spesso contraddittorie. A proposito d'una tale proposta teorica, vorrei limitarmi a una sola osservazione. Da una parte Orlando era pronto ad accogliere nella sua teoria contributi diversi (in particolare sarà decisivo per lui il pensiero di Ignacio Matte Blanco e la sua concezione dell'inconscio come «pensiero bilogico»), con uno spirito sempre aperto al nuovo, da esploratore. Dall'altra egli era estraneo a ogni concessione all'eclettismo che derogasse dalla coerenza più rigorosa, e in tutta la sua vita, in particolare, ha sempre mostrato una sovrana indifferenza nei confronti delle mode.

I volumi teorici, nonostante l'editore Einaudi abbia da un certo momento in poi mostrato disinteresse per la critica letteraria e quindi anche verso di essi, hanno conosciuto una grande fortuna italiana, testimoniata dalle numerose ristampe. Sono anche stati parzialmente tradotti negli Stati Uniti. Resistenze ben più forti, invece, hanno incontrato presso la cultura francese, dovute a incomprensioni e refrattarietà che hanno profondamente amareggiato l'ultima parte della vita di Orlando. Dopo il ciclo teorico, egli si è dedicato a quello che secondo molti costituisce il suo capolavoro: Gli oggetti desueti nelle immagini della letteratura (1993). Opera grandiosa in cui confluiscono letture sterminate degne di uno studioso d'altra epoca che la nostra, dalla Bibbia a García Márquez passando per l'intera letteratura occidentale. Una riflessione teorica rigorosa gli consente di ordinare questo immenso materiale in dodici categorie dalla rilevanza analitica e storica sorprendente. Il volume, già tradotto nel 
2006 presso Yale University Press, sta per apparire in Francia presso la casa editrice Classiques Garnier, anche grazie alle sollecitazioni di prestigiosi intellettuali francesi che si sono finalmente mostrati sensibili all'originalità e allo spessore d'un simile lavoro. Purtroppo, Orlando è morto proprio mentre stava correggendo le bozze di questo volume d'imminente pubblicazione.

L'ultima fase dell'attività di Francesco Orlando sembra riallacciare i legami con i suoi inizi. Nel 1998 egli pubblica L'Intimità e la Storia, una lettura illuminante del Gattopardo nella quale viene dimostrato il carattere europeo di questo capolavoro in polemica con le letture «regionaliste» dominanti nella cultura italiana. Soprattutto, pubblica un suo romanzo scritto a vent'anni e riscritto a settanta, La doppia seduzione (Einaudi, 2010), storia di un'educazione sentimentale fallita di due giovani legati da un rapporto sado-masochistico, in una città del sud, che si conclude tragicamente. Il romanzo, sorretto da una ricerca stilistica di eccezionale sapienza, è animato anche da un intento militante: ribadire la concezione freudiana della fondamentale bisessualità umana contro ogni reificazione dell'omosessualità come terzo sesso. Ormai in pensione dal 2009, era in piena attività quando è stato sorpreso dalla morte il 22 giugno 2010. Aveva in cantiere ben quattro volumi: uno teorico su quelle che chiamava le figure dell'invenzione; uno sul soprannaturale di cui il fantastico è concepito come una piccola parte; uno, di grande portata antropologica e non solo letteraria, sulla figura del Cornuto dall'antichità classica alla letteratura moderna; e infine uno su Wagner, sicuramente il più grande amore 'artistico' della sua vita, l'autore in cui si è più identificato e la cui opera ha segnato con maggiore profondità la sua riflessione. La gentilezza d'altri tempi, il senso della giustizia e del merito - oltre al prestigio scientifico - hanno continuato a procurare a Francesco Orlando non solo il rispetto ma anche l'amicizia di tanti studiosi che pure non si erano formati con lui. Era rimasto il punto di riferimento scientifico ed esistenziale dei numerosi allievi che sono tutti orgogliosi di essere stati alla sua scuola. E, soprattutto, era ancora circondato, come durante tutta la sua vita, dalla dedizione di giovani che trovavano in lui un maestro entusiasta, attento, paziente ${ }^{1}$.

FRANCESCO FIORENTINO

${ }^{1}$ Ricordiamo con commozione che Francesco Orlando fu responsabile per qualche anno, con
Arnaldo Pizzorusso, della sezione bibliografica settecentesca di «Studi Francesi». (N.d.R.) 